archives-ouvertes

\title{
Prohibitin Inactivation in Adipocytes Results in Reduced Lipid Metabolism and Adaptive Thermogenesis Impairment
}

Zhanguo Gao, Alexes Daquinag, Cale Fussell, Amel Djehal, Laurent Désaubry, Mikhail Kolonin

\section{To cite this version:}

Zhanguo Gao, Alexes Daquinag, Cale Fussell, Amel Djehal, Laurent Désaubry, et al.. Prohibitin Inactivation in Adipocytes Results in Reduced Lipid Metabolism and Adaptive Thermogenesis Impairment. Diabetes, American Diabetes Association, 2021, 70 (10), pp.2204-2212. 10.2337/db21-0094 . hal-03400777

\section{HAL Id: hal-03400777 https://hal.archives-ouvertes.fr/hal-03400777}

Submitted on 25 Oct 2021

HAL is a multi-disciplinary open access archive for the deposit and dissemination of scientific research documents, whether they are published or not. The documents may come from teaching and research institutions in France or abroad, or from public or private research centers.
L'archive ouverte pluridisciplinaire HAL, est destinée au dépôt et à la diffusion de documents scientifiques de niveau recherche, publiés ou non, émanant des établissements d'enseignement et de recherche français ou étrangers, des laboratoires publics ou privés. 


\title{
Prohibitin Inactivation in Adipocytes Results in Reduced Lipid Metabolism and Adaptive Thermogenesis Impairment
}

\author{
Zhanguo Gao, ${ }^{1}$ Alexes C. Daquinag, ${ }^{1}$ Cale Fussell, ${ }^{1}$ Amel Djehal, ${ }^{2,3}$ Laurent Désaubry, ${ }^{2}$ \\ and Mikhail G. Kolonin ${ }^{1}$
}

${ }^{1}$ The Brown Foundation Institute of Molecular Medicine, University of Texas Health Science Center, Houston, TX 77030, USA

2 INSERM-University of Strasbourg, Regenerative Nanomedicine Laboratory (UMR1260), Faculty of Medicine, FMTS, Strasbourg, France

${ }^{3}$ Superior National School Biotechnology Taoufik Khaznadar, Constantine, Algeria

Corresponding author: Mikhail G. Kolonin

mikhail.g.kolonin@uth.tmc.edu

Phone: $713-500-3146$ 
Prohibitin-1 (PHB) is a multifunctional protein previously reported to be important for adipocyte function. PHB is expressed on the surface of adipose cells, where it interacts with a long chain fatty acid (LCFA) transporter. Here, we show that mice lacking PHB in adipocytes (PHB Ad-KO) have a defect in fat tissue accumulation despite having larger lipid droplets in adipocytes due to reduced lipolysis. Although PHB Ad-KO mice do not display glucose intolerance, they are insulin resistant. We show that PHB Ad-KO mice are lipid intolerant due to a decreased capacity of adipocytes for LCFA uptake. Instead, PHB Ad-KO mice have increased expression of glucose transporter GLUT1 in various tissues and use glucose as a preferred energy source. We demonstrate that PHB Ad-KO mice have defective brown AT, are cold-intolerant, and display a reduced basal energy expenditure. Systemic repercussions of PHB inactivation in adipocytes were observed in both males and females. Consistent with lower cellular mitochondrial content and reduced UCP1 protein expression, brown adipocytes lacking PHB display decreased proton leak and switch from aerobic metabolism to glycolysis. Treatment of differentiating brown adipocytes with small molecules targeting PHB suppressed mitochondrial respiration and uncoupling. Our results demonstrate that PHB in adipocytes is essential for normal fatty acid uptake, oxidative metabolism, and adaptive thermogenesis. We conclude that PHB inhibition could be investigated as an approach to altering energy substrate utilization. 


\section{INTRODUCTION}

An important function of adipose tissue (AT) is the maintenance of energy balance and healthy metabolism (1). Adipocytes are the lipid-storing cells of AT, dysfunction of which results in type-2 diabetes. Prohibitin-1 (PHB) is a multi-functional protein found in various cellular compartments and also secreted, reported to be important for normal adipocyte function (2). Studies based on small molecule ligands of PHB modifying its function have helped to map the diverse functions of this protein in metabolic, inflammatory, cardiac, connective tissue, and neurological diseases, as well as in cancer (3). A key insight on adipose function of PHB was the mouse model overexpressing PHB from the aP2 promoter, which resulted in increased adiposity and impaired glucose homeostasis despite elevated mitochondrial biogenesis (4). By screening for molecules that home to white AT (WAT), we previously identified a peptide (sequence KGGRAKD) that binds to PHB on the surface of adipose endothelium (5). In search for PHB function in WAT endothelium, we have identified annexin A2 (ANX2), as a PHB-binding protein containing the amino acid sequence KGRRAED mimicked by the WAT-homing peptide KGGRAKD. We have shown that ANX2 and PHB are found in a protein complex present in the cell membrane lipid rafts of both mouse and human WAT (6). We subsequently demonstrated that PHB and ANX2 are found in complex with the fatty acid translocase CD36 and demonstrated that the ANX2-PHB interaction supports LCFA uptake by adipocytes in cell culture (7). However, the importance of PHB in adipose physiology still remained unclear due to the lack on in vivo adipocyte loss-offunction models.

The ability of adipocytes in WAT to store and metabolize lipids predetermines susceptibility to metabolic diseases (1). Visceral AT (VAT) mainly stores lipids, and its excessive expansion, inflammation, and dysfunction in obesity is linked with the metabolic syndrome (8). In contrast, subcutaneous AT (SAT) can protect from metabolic disease through generation of mitochondriarich adipocytes specialized to activate lipolysis and burn lipids through adaptive thermogenesis 
(9). Like brown AT (BAT), beige adipocytes in SAT express the uncoupling protein 1 (UCP1), which leaks protons to uncouple substrate oxidation from ATP synthesis (10). Both BAT and beige adipocytes activate energy expenditure and can counteract metabolic consequences of obesity in mice (1). A possible function of PHB in BAT has not been investigated.

Based on our previous findings (7), we hypothesized that PHB promotes LCFA uptake in WAT. In addition, based on the known mitochondrial function of PHB (4), we also hypothesized that it regulates metabolic activity and may be particularly important for BAT function. Here, we tested these hypotheses by analyzing mice lacking PHB specifically in adipocytes and by using pharmacological PHB modulators.

\section{RESEARCH DESIGN AND METHODS}

\section{Animal Experiments}

All animal experimentations were approved by the University of Texas Health Animal Care and Use Committee. Mice were housed in the animal facility with a $12 \mathrm{~h}$ light/dark cycle at temperature of $22-24^{\circ} \mathrm{C}$ with free access to water and diet. PHB fl/fl mice (11) and Apn-Cre mice (Jackson Laboratories Stock 010803) were used for crosses. For DIO induction, mice were fed 58 kcal\% (fat) diet (Research Diets, D12331). Body composition was measured using EchoMRI-100T (Echo Medical Systems). Indirect calorimetry studies were performed with OXYMAX (Columbus Instruments) Comprehensive Lab Animal Monitoring System (CLAMS) as described previously (12). Food intake and spontaneous locomotor activity was quantified over a 2-day time course at the same time. The core body temperature was determined using a MicroTherma $2 \mathrm{~K}$ High Precision Type K Thermocouple Meter (THS-221-092, ThermoWorks)/RET-3 rectal probe (Braintree Scientific) as described previously (12). Cold tolerancel adaptive thermogenesis was measured upon placing mice into environmental chamber IS33SD (Powers Scientific) as 
described (13). For glucose tolerance test, glucose ( $2 \mathrm{~g} / \mathrm{kg}$ body weight) was injected i.p. into overnight-fasted mice. For insulin tolerance test, insulin (0.6 U/kg body weight) was injected i.p. into mice fasted four hours. Blood glucose concentration was measured with a glucometer (One Touch Ultra). Intravenous fat tolerance tests were performed by injecting overnight-fasted mice with $100 \mu \mathrm{L}$ of Intralipid $20 \%$ fat emulsion. Blood from tail vain was measured for triglyceride using EnzyChrom ${ }^{\text {TM }}$ Triglyceride Assay Kit (BioAssay System, cat \# ETGA-200). Lipolysis was induced by i.p. injection of isoproterenol $(10 \mathrm{mg} / \mathrm{kg})$. Plasma FFA were measured with a kit from BioAssay System (EnzyChromTM Free Fatty Assay Kit, cat \# EFFA-100).

\section{Cell lines and Culture assays}

For stromal cell isolation, outer ears and SAT pads of 8-week-old mice were excised, minced and digested in $0.5 \mathrm{mg} / \mathrm{ml}$ collagenase type I (Worthington Biochemical) and $2.5 \mathrm{mg} / \mathrm{ml}$ of dispase Roche, cat \# 04942078001 ) solution in a shaking bath for $1 \mathrm{~h}$ at $37^{\circ} \mathrm{C}$. The cell suspension was filtered through a $70 \mu \mathrm{m}$ cell strainer (Thomas Scientific, cat \# 1181X53) followed by centrifugation $(360 \times \mathrm{g}, 5 \mathrm{~min}, \mathrm{RT})$. Pelleted cells were plated in $100 \mathrm{~mm}$ Petri dishes $(p=0)$ in DMEM / 10\% FBS. Brown preadipocyte cells (13) were cultured in DMEM / 10\% FBS. For white adipogenesis induction, cells grown to confluence were cultured in medium containing $1.7 \mu \mathrm{M}$ insulin/0.5 mM IBMX, $1 \mu \mathrm{M}$ dexamethasone and $5 \mu \mathrm{M}$ pioglitazone for 3 days and $1.7 \mu \mathrm{M}$ insulin afterwards, as described previously (12). For brown adipogenesis induction, cells grown to confluence were cultured in medium containing $50 \mathrm{nM}$ insulin/0.5 mM IBMX, $1 \mu \mathrm{M}$ dexamethasone, 1 nM 3,5,3'Triiodothyronine (T3) and $5 \mu \mathrm{M}$ rosiglitazone for 3 days, and $50 \mathrm{nM}$ insulin with $1 \mathrm{nM}$ T3 afterwards. For fatty acid uptake, adipocytes from differentiated ear fibroblast cells were treated with $2 \mu \mathrm{M}$ BODIPY-C16 for 30 min, fixed and stained with Hoechst 33258 (Invitrogen, H3569), and imaged as described (7). LCFA uptake by adipocytes was quantified with the QBT TM Assay (Molecular Devices) as described (7). PHB ligands $(3 ; 14 ; 15)$ were diluted as described previously (JI130 at $0.1 \mathrm{uM}$, Mel56 at $10 \mathrm{uM}, \mathrm{SA} 1 \mathrm{~m}$ at $10 \mathrm{uM})$ in beige adipocyte inducing medium. 
The Seahorse XF Cell Mito Stress Test Kit (Agilent Technologies, Cat \# 103015-100 ) was used to analyze mitochondrial respiration in beige adipocytes treated with PHB inhibitors for 2 hours. Oxygen consumption rate (OCR) was measured upon successive treatment with oligomycin (1mM), FCCP (Carbonyl cyanide-p-trifluoromethoxyphenylhydrazone, 1mM) and rotenone / antimycin $A(0.5 \mathrm{mM})$.

\section{Quantitative real-time RT-PCR}

Total RNA was extracted using the Trizol Reagent (Life Technologies, 15596018). Complementary DNAs were generated using High-Capacity cDNA Reverse Transcription Kit (Applied Biosystems, 4368814). PCR reactions were performed on a CFX96 Real-Time System C1000 Touch thermal cycler (Bio-Rad) using Q-PCR Master Mix (Gendepot, Q5600-005). Expression of mouse Phb, Ucp1, and Cox IV was normalized to 18S RNA. Primers were as follows: Ucp 1, 5'-TCTCAGCCGGCTTAATGACTG-3' and 5'-GGCTTGCATTCTGACCTTCAC-3'; Cox IV, 5'- CtgCCCGGAGTCTGGTAATG-3' and 5'- CAGTCAACGTAGGGGGTCATC-3'; Phb, 5'- GCATTGGCGAGGACTATGAT-3' and 5'- CTCTGTGAGGTCATCGCTCA-3' and 18S RNA, 5'-AAGTCCCTGCCCTTTGTACACA-3' and 5'-GATCCGAGGGCCTCACTAAAC-3'.

\section{Cell and tissue analysis}

Paraformaldehyde-fixed cells and formalin-fixed paraffin-embedded tissue sections were analyzed by immunofluorescence as described previously (12). Upon blocking, primary $\left(4^{\circ} \mathrm{C}, 12\right.$ h) and secondary (room temperature, $1 \mathrm{~h}$ ) antibody incubations were carried out. Antibodies, diluted in PBS with $0.05 \%$ Tween 20, were as follows: anti-UCP1 (Alpha Diagnostic, UCP-11A; 1:400); anti-PHB (Invitrogen, cat \# MA5-32000, 1:100); anti-Perilipin1 (Abcam, cat \# ab61682; 1:200) and pHSL (Cell Signaling, cat \# 4126,1:100). Donkey Alexa 488-conjugated (1:200) and Cy3-conjugated (1:300) IgG, were from Jackson ImmunoResearch. MitoTracker® Deep Red 
(ThermoFisher, cat\# M22426) was used (200nM) to stain live cells for 30 minutes prior to fixation. Nuclei were stained with Hoechst 33258 (Invitrogen, H3569). Images were acquired with a confocal Leica TCS SP5 microscope/LAS AF software (Leica) or Carl Zeiss upright Apotome Axio Imager Z1/ZEN2 Core Imaging software. Adipocyte size was quantified by measuring cell area with Adiposoft plugin of Image J software.

\section{Immunoblotting}

Whole-cell lysates was prepared in RIPA buffer and analyzed as described previously (12). AT lysates were separated by SDS-PAGE and subsequently analyzed by immunoblotting. The following antibodies were used: anti-UCP1 (Sigma, U6382; 1:5000), anti-PHB (Invitrogen, cat \# MA5-32000, 1:1000), and anti- $\beta$-actin (Abcam, ab8226; 1:5000). The signal was detected using the Odyssey CLx imaging system (LI-COR) and quantified with Image $\mathrm{J}$ analysis software.

\section{Statistical Analysis}

All statistical analyses were performed with GraphPad Prism 6 software. Experimental results are shown as mean +/- SEM. Two-tailed Student unpaired t tests were performed to littermates unless otherwise indicated. $\mathrm{P}<0.05$ was considered significant.

Data Sharing Statement: additional data and critical resources supporting the reported findings, methods, and conclusions will be available upon request.

\section{RESULTS}

\section{Adipose tissue abnormality in mice with PHB-KO adipocytes}

To create a model with a knock-out (KO) of PHB in adipocytes, we used an Adiponectin-Cre (ApnCre) strain with adipocyte-specific Cre expression (16). We crossed it with the Phb1 ${ }^{\text {fl/fl }}$ strain (11) 
in which the Phb gene is flanked by loxP recombination sites. Apn-Cre;Phb fl/fl (PHB adipo-KO) and Cre-negative Phbl/fl (WT) littermates were then compared. PHB knockout was confirmed by western blot on extracts from WAT and BAT, which demonstrated a marked reduction in protein expression (Fig. 1A). Residual expression remained as expected due to PHB expression in other cell types (stroma, endothelium and leukocytes). We then phenotyped male PHB adipo-KO and WT littermates. EchoMRI analysis demonstrated a reduced fat body mass accumulation in KO mice (Fig.1B), which by 3 months of age was detectable for males and females (Fig.1 A). While high fat diet (HFD) feeding expectedly induced obesity development in WT mice (Fig.1 B), the KO littermates failed to increase adiposity (Fig. 1C). Analysis of internal organs demonstrated both subcutaneous and visceral lipodystrophy in PHB Ad-KO males (Fig. 1D) and females (Fig. S1B). There was no significant effect of PHB adipo-KO on lean body mass (Fig. 1C), as well as on appetite (Fig. S1C) or locomotor activity (Fig. S1D). Analysis of haematoxylin / eosin (H\&E)stained WAT sections revealed that PHB Ad-KO mice have significantly larger adipocytes in both VAT and SAT, compared to WT littermates (Fig. 1E; Fig. S1E). To investigate how PHB loss affects lipid droplets, we induced adipogenesis in stromal cells isolated from mouse SAT and ears. Lipid-laden adipocytes differentiated from cells of both WT and PHB Ad-KO mice (Fig. S1F), and there was no difference in adipogenesis marker expression (Fig. S1G). However, consistent with a previous report (17), knockout of Phb gene in 3T3-L1 preadipocytes (Fig. S1H) resulted in an inhibition of adipogenic transcription program (Fig. S1I) and delayed lipid droplet accumulation (Fig. S1J). This indicates that, while PHB regulates adipogenesis, its deletion in mature adipocytes PHB Ad-KO mice does not preclude lipid droplet maintenance. Consistent with WAT adipocyte hypertrophy, cultured PHB Ad-KO adipocytes, confirmed to lack PHB by immunofluorescence $(F L)$, accumulated lipid droplets that tended to be larger than in WT adipocytes (Fig. 1F). This indicated that PHB also plays a role in lipid droplet maintenance postadipogenesis. 


\section{Lipid metabolism in PHB ad-KO mice}

To find an explanation to the WAT phenotype of PHB Ad-KO mice, we analyzed lipid metabolism. First we analyzed cultured adipocytes for the ability to uptake a fluorophore-labeled palmitic acid, BODIPY-C ${ }_{16}$ FA. This revealed a marked defect of PHB-null adipocytes to uptake LCFA (Fig. 2A). Quantification of this defect was performed by the QBT assay with a fluorophore-labeled fatty acid. This conformed efficiency of insulin-induced LCFA uptake by PHB-null adipocytes (Fig. 2B). To confirm these findings in vivo, we performed a lipid tolerance test on mice. Upon iv LCFA infusion, the time course of triglyceride blood concentration demonstrated a delay in clearance for PHB Ad-KO littermates compared to WT (Fig. 2C). Consistent with reduced LCFA uptake, indirect calorimetry analysis of mice in metabolic chambers demonstrated that PHB Ad-KO mice had increased respiratory exchange ratio (RER) (Fig. 2D), indicating lower utilization of lipid as energy source. Non-fasting circulating FFA levels were found to be higher in PHB Ad-KO littermates, consistent with a decreased ability of adipocytes to uptake and utilize them (Fig. 2E). Liver histopathology revealed steatosis in PHB-Ad KO mice (Fig. S2A). Triglyceride content was also quantified and found significantly higher for both liver and skeletal muscle of PHB-Ad KO mice (Fig. S2B). This indicates that inefficient adipocyte fatty acid uptake in these mice results in increased ectopic lipid deposition.

As a potential mechanistic explanation for the increased adipocyte size despite a decrease in LCFA uptake, we investigated lipolysis. Upon its induction by injection of a $\beta$-adrenergic agonist isoproterenol, circulating FFA levels were expectedly increased in WT mice. In contrast to WT mice, the induction by isoproterenol was not observed in PHB Ad-KO littermates (Fig. 2E). Consistent with this, phosphorylated (activated) hormone-sensitive lipase (pHSL) expression was virtually undetectable in both white and brown adipocytes of PHB Ad-KO mice (Fig. 2F). Because HSL is phosphorylated by protein kinase A (PKA), we measured PKA activity with phospho-PKA substrate antibodies. Our data indicate relatively low PKA activity in SAT, VAT, and BAT of PHB- 
KO AT mice, elucidating the mechanism for reduced lipolysis (Fig. S2C). This indicates that PHB$\mathrm{KO}$ adipocyte hypertrophy results from a lipolysis defect.

\section{Glucose metabolism in PHB ad-KO mice}

RER increase observed in male (Fig. 2D) and female (Fig. S3A) PHB Ad-KO mice suggested their switch to glucose as the main energy substrate. In accord with this, IF analysis expression of a glucose transporter GLUT1 was found to be higher in WAT and BAT, as well as in liver and skeletal muscle of PHB Ad-KO mice (Fig. S3B). There was a trend for higher non-fasting glucose levels in PHB Ad-KO male littermates, suggesting an increase in gluconeogenesis (Fig. S3C). Analysis of male mice raised on chow did not reveal a difference in fasting glucose clearance rates (Fig. S3D). Analysis of PHB Ad-KO females raised on chow also revealed increased steadystate glucose levels and normal fasting glucose clearance (Fig. S3F-G). Interestingly, upon HFD feeding PHB Ad-KO mice displayed a higher glucose tolerance than WT littermates (Fig. 2G). However, both male and female PHB Ad-KO littermates displayed a significantly lower insulin tolerance when raised not only on HFD (Fig. 2H) but also on chow (Fig. S3E; Fig. S3H). Combined, our data indicate that adipocyte PHB deficiency leads to systemic upregulation of glucose metabolism and insulin resistance.

\section{Brown AT and adaptive thermogenesis in PHB ad-KO mice}

Thermogenic activity of BAT relies on both lipolysis and mitochondrial function. Based on the lipolysis defect revealed here (Fig. 2E-F) and previous $\mathrm{PHB}$ implication in mitochondrial biogenesis (4), we analyzed BAT. Interscapular AT was drastically smaller in both male and female PHB Ad-KO mice, compared to WT littermates (Fig. 3A). Its WAT-like appearance indicated a lower mitochondrial content. H\&E-staining of interscapular AT sections confirmed its whitening in PHB Ad-KO mice (Fig. 3B), with larger lipid droplets being consistent with deficient lipolysis. Western blotting of protein extracts demonstrated that UCP1 expression in BAT of PHB 
Ad-KO mice was undetectable (Fig. 3C). As expected for the lack of this key uncoupling protein, core body temperature measurement in mice placed at $4 \mathrm{C}$, indicated a significantly reduced cold tolerance of PHB Ad-KO males (Fig. 3D) and females (Fig. S4A). Consistent with this, oxygen consumption measured by indirect calorimetry revealed a reduction nighttime and daytime energy expenditure in PHB Ad-KO males (Fig. 3E) and females (Fig. S4B).

\section{Mitochondrial dysfunction in PHB-KO adipocytes}

In addition to UCP1 expression loss in BAT, RT-PCR analysis revealed lower Cox IV gene expression in PHB Ad-KO BAT and WAT (Fig. S4C). Reduction in this nuclear-encoded enzyme essential for electron transport may account for mitochondrial dysfunction. In addition, there was a significant reduction of mitochondrial content in both BAT and WAT (Fig. S4D). We used a cell culture model to investigate the consequences of mitochondrial defect in PHB-KO adipocytes. UCP1 expression was observed in WT but not in PHB-KO brown adipocytes differentiated from stromal cells (Fig. 3F-G). Notably, differentiated PHB-KO cells had larger lipid droplets, indicative of them differentiating into white rather than brown adipocytes (Fig. 3F-G). The Seahorse XF Cell Mito Stress Assay was then performed on these cells to analyze mitochondrial respiration. Lower basal and induced oxygen consumption rate (OCR) revealed a defect in mitochondrial respiration of PHB-KO adipocytes (Fig. 3H). Consistent with the lack of UCP1, oligomycin-resistant OCR was lower in PHB-KO adipocytes, indicating a decreased proton leak (Fig. 3I). Importantly, extracellular acidification rate (ECAR), measured by the XF Cell Mito Stress Assay, was found to be significantly higher for PHB-KO adipocytes (Fig. S4E). These data indicate that the cells switch from mitochondria-dependent oxidative phosphorylation to glycolysis in the absence of PHB.

\section{Pharmacological PHB targeting block with brown adipocyte differentiation and function}

Finally, we investigated the involvement of PHB in different aspects of brown adipocyte biology. We used mouse immortalized brown preadipocytes (IBP) (13) induced to undergo brown adipogenesis. For PHB targeting, we used the previously characterized PHB small molecule 
ligands JI130, Mel56, and SA1m $(3 ; 14 ; 15)$. Treatment of preadipocytes during adipogenesis induction did not interfere with differentiation, as evident from lipid droplet formation (Fig. 4A). However, MitoTracker staining revealed a notably lower signal in cells treated with each PHB ligand, compared to control adipocytes (Fig. 4A). This indicated that PHB is important for mitochondrial biogenesis in brown adipocytes. Consistent with this, Seahorse Real-time ATP Rate Assay demonstrated a decrease in mitochondrial respiration (Fig. S5A) and in extracellular acidification (Fig. S5B), with JI130 having the strongest effect compared to the other two PHB small molecules. There was also a shift to glycolytic ATP production (Fig. S5C) and a decrease in uncoupled respiration (Fig. S5D) in treated cells. We then treated IBP-derived adipocytes with the same PHB modulators after the completion of brown differentiation. The Seahorse XF Cell Mito Stress Assay revealed that J1130 strongly decreased both basal and induced OCR, while Mel56 and SA1m also had lower effects (Fig. 4B). All three compounds significantly decreased proton leak, as well as spare respiration capacity (Fig. 4C). However, neither compound significantly affected ATP production, confirming that cells switch from mitochondria-dependent oxidative phosphorylation to glycolysis in the absence of PHB.

\section{DISCUSSION}

Our analysis of PHB adipo-KO mice reveals their defect in fat mass accumulation despite normal food intake. Adipocyte hypoplasia accounts for this lipodystrophy because adipocyte size is increased in WAT of PHB adipo-KO mice. While we confirm the previously reported (17) requirement of PHB for initiation of adipogenesis, in PHB adipo-KO mice adipocytes differentiate normally because PHB deletion is driven by expression of $A p n$, a late adipogenesis gene. Our data show that inactivation of PHB post-adipogenesis leads to hypertrophy of mature adipocytes due to a reduction in PKA and HSL activity resulting in decreased lipolysis. This could be explained by reduced activity of adrenergic receptors in "whitened" PHB-deficient adipocytes because PKA activity is regulated by cAMP, downstream of GPCR activation. Although PHB Ad- 
KO mice do not display glucose intolerance, both males and females have incresed levels of blood glucose and are insulin resistant. Diabetes development in these mice occurs despite a systemic increase in glucose transprter expression. Our results indicate that the drastic disbalance in substrate utilization in PHB Ad-KO mice is due to their lipid intolerance. This is supported by the notion that PHB Ad-KO mice fed HFD clear glucose better than WT mice, suggesting that increased lipid load further boosts glucose utilization. Lipid intolerance of PHB Ad-KO mice, resulting in steatosis, could be explained by a decreased capacity of adipocytes for LCFA uptake, LCFA oxidation, or both. By using cell culture models, we demonstrate a defect in lipid uptake by PHB-KO adipocytes. This is consistent with the phenotypes reported for CD36 KO (18) and ANX2 KO (7) mice. Our data confirms that PHB, in complex with CD36, regulates FA uptake and contributes to lipid accumulation in AT.

As expected, the metabolic consequences of adipocyte PHB overexpression (4) are opposite to the phenotype we describe in PHB Ad-KO mice. Importantly, our data reveals a previously unappreciated function of PHB in brown adipocytes, pointing to the role of PHB in fatty acid oxidation. A marked defect in BAT observed in PHB Ad-KO mice results in their cold-intolerance and reduced energy expenditure. The reduced mitochondrial content in AT of PHB Ad-KO mice is consistent with our results from PHB-KO cells, as well as WT cells treated with PHB ligands. The apparent role of PHB in mitochondrial biogenesis and function is consistent with results from PHB overexpression models (4). We show that, brown adipocytes lacking PHB have a decreased proton leak, consistent with a reduced UCP1 protein expression. PHB-null cells also have lower mitochondrial respiration, consistent with a reduced expression of mitochondrial transporter Cox IV. Upon treatment of differentiated brown adipocytes with pharmacological PHB modulators, a decrease in mitochondrial respiration and uncoupling was similarly observed. Importantly, glycolysis was induced in PHB-KO or PHB-inhibited adipocytes in compensation for the oxidative defect. Combined, our results demonstrate that PHB in adipocytes is essential for normal lipid 
metabolism and adaptive thermogenesis, and that its adipocyte dysfunction derails AT function, energy substrate utilization, and leads to metabolic dysfunction.

Our previous studies discovered that PHB is expressed on the surface of adipocytes and endothelial cells selectively in AT. We have used a PHB-homing peptide, KGGRAKD, to direct an apoptosis-inducing peptide ${ }_{\mathrm{D}}(\mathrm{KLAKLAK})_{2}$ to WAT in an experimental approach to obesity reversal (5). WAT vascular-targeting capacity of the hunter-killer CKGGRAKDC-D(KLAKLAK) ${ }_{2}$ peptide has been validated in mouse, rat, and non-human primates, has shown to have anti-obesity effects $(19 ; 20)$. This study suggests a possibility that the anti-obesity effect could result in part due to the loss of PHB function executed by cells expressing it in adipose tissues.

Acknowledgments. The authors thank Shelly Lu for providing Phb1 $1^{\mathrm{fl} / \mathrm{fl}}$ mice.

Funding. This work was supported by NIH grant 2R01DK088131 to MGK.

Duality of Interest. No potential conflicts of interest relevant to this article were reported.

Authors Contributions. M.G.K. Z.G. and L.D. conceived / designed the experiments, analyzed data, and wrote the manuscript; Z.G., A.C.D., A.D. and C.F. designed and performed the experiments, analyzed data, and edited the manuscript. M.G.K. is the guarantor of this work and, as such, had full access to all the data in the study and takes responsibility for the integrity of the data and the accuracy of the data. Guarantor: M.G.K.

\section{References}

1. Rosen ED, Spiegelman BM: What we talk about when we talk about fat. Cell $2014 ; 156: 20-44$

2. Ande SR, Nguyen KH, Nyomba BLG, Mishra S: Prohibitin in Adipose and Immune Functions. Trends Endocrinol Metab 2016;27:531-541 
3. Wang D, Tabti R, Elderwish S, Abou-Hamdan H, Djehal A, Yu P, Yurugi H, Rajalingam K, Nebigil CG, Desaubry L: Prohibitin ligands: a growing armamentarium to tackle cancers, osteoporosis, inflammatory, cardiac and neurological diseases. Cell Mol Life Sci 2020;77:3525-3546

4. Ande SR, Nguyen KH, Padilla-Meier GP, Wahida W, Nyomba BL, Mishra S: Prohibitin overexpression in adipocytes induces mitochondrial biogenesis, leads to obesity development, and affects glucose homeostasis in a sex-specific manner. Diabetes 2014;63:3734-3741

5. Kolonin MG, Saha PK, Chan L, Pasqualini R, Arap W: Reversal of obesity by targeted ablation of adipose tissue. Nature Med 2004;10:625-632

6. Staquicini FI, Cardo-Vila M, Kolonin MG, Trepel M, Edwards JK, Nunes DN, Sergeeva A, Efstathiou E, Sun J, Almeida NF, Tu SM, Botz GH, Wallace MJ, O'Connell DJ, Krajewski S, Gershenwald JE, Molldrem JJ, Flamm AL, Koivunen E, Pentz RD, Dias-Neto E, Setubal JC, Cahill DJ, Troncoso P, Do KA, Logothetis CJ, Sidman RL, Pasqualini R, Arap W: Vascular ligand-receptor mapping by direct combinatorial selection in cancer patients. Proc Natl Acad Sci USA 2011;108:18637-18642

7. Salameh A, Daquinag AC, An Z, K. H, Pasqualini R, Arap W, Kolonin MG: Prohibitin/annexin 2 interaction regulates fatty acid transport in adipose tissue. JCI Insight 2016;1:86351-86357

8. Sun K, Tordjman J, Clement K, Scherer PE: Fibrosis and adipose tissue dysfunction. Cell Metab $2013 ; 18: 470-477$

9. Kajimura S, Spiegelman BM, Seale P: Brown and Beige Fat: Physiological Roles beyond Heat Generation. Cell Metab 2015;22:546-559

10. Cannon B, Nedergaard J: Brown adipose tissue: function and physiological significance. Physiol Rev 2004;84:277-359

11. Ko KS, Tomasi ML, Iglesias-Ara A, French BA, French SW, Ramani K, Lozano JJ, Oh P, He L, Stiles BL, Li TW, Yang H, Martinez-Chantar ML, Mato JM, Lu SC: Liver-specific deletion of prohibitin 1 results in spontaneous liver injury, fibrosis, and hepatocellular carcinoma in mice. Hepatology $2010 ; 52: 2096-2108$ 
12. Gao Z, Daquinag AC, Fussell C, Zhao Z, Dai Y, Rivera A, Snyder BE, Eckel-Mahan KL, Kolonin MG: Age-associated telomere attrition in adipocyte progenitors predisposes to metabolic disease. Nat Metab 2020;2:1482-1497

13. Gao Z, Daquinag AC, Su F, Snyder B, Kolonin MG: PDGFRalpha / PDGFRbeta signaling balance modulates progenitor cell differentiation into white and beige adipocytes. Development $2018 ; 145: 1-13$

14. Djehal A, Krayem M, Najem A, Hammoud H, Cresteil T, Nebigil CG, Wang D, Yu P, Bentouhami E, Ghanem GE, Desaubry L: Targeting prohibitin with small molecules to promote melanogenesis and apoptosis in melanoma cells. Eur J Med Chem 2018;155:880-888

15. Perron A, Nishikawa Y, Iwata J, Shimojo H, Takaya J, Kobayashi K, Imayoshi I, Mbenza NM, Takenoya M, Kageyama R, Kodama Y, Uesugi M: Small-molecule screening yields a compound that inhibits the cancer-associated transcription factor Hes1 via the PHB2 chaperone. The Journal of biological chemistry 2018;293:8285-8294

16. Eguchi J, Wang X, Yu S, Kershaw EE, Chiu PC, Dushay J, Estall JL, Klein U, Maratos-Flier E, Rosen ED: Transcriptional control of adipose lipid handling by IRF4. Cell Metab 2011;13:249-259 17. Ande SR, Xu Z, Gu Y, Mishra S: Prohibitin has an important role in adipocyte differentiation. Int J Obes 2012;36:1236-1244

18. Febbraio M, Abumrad NA, Hajjar DP, Sharma K, Cheng W, Pearce SF, Silverstein RL: A null mutation in murine CD36 reveals an important role in fatty acid and lipoprotein metabolism. J BiolChem 1999;274:19055-19062

19. Barnhart KF, Christianson DR, Hanley PW, Driessen WH, Bernacky BJ, Baze WB, Wen S, Tian M, Ma J, Kolonin MG, Saha PK, Do KA, Hulvat JF, Gelovani JG, Chan L, Arap W, Pasqualini R: A peptidomimetic targeting white fat causes weight loss and improved insulin resistance in obese monkeys. Sci Transl Med 2011;3:108-112

20. Kim DH, Woods SC, Seeley RJ: Peptide designed to elicit apoptosis in adipose tissue endothelium reduces food intake and body weight. Diabetes 2010;59:907-915 


\section{Figure Legends}

Figure 1- Adipocyte PHB KO mice are lipodystrophic despite adipocyte hypertrophy. A: Western blotting on extracts from WAT and BAT confirms the loss of PHB expression in PHB Ad-KO male mice, as compared to WT littermates. Actin immunoblotting: loading control. Graph: band intensity quantification. B: EchoMRI measurements on male mice raised for 3 months on chow and 3 months of HFD reveals reduced fat mass accumulation in PHB Ad-KO littermates. $\mathrm{N}=5$ mice. $C$ : EchoMRI measurements on male mice from $B$ show a lack of difference in lean body mass. $D$ : Images demonstrating subcutaneous and visceral lipodystrophy in PHB Ad-KO male littermates. Resected SAT and VAT (one side) are compared on the right for males and females. E: H\&Estaining of sections reveals larger adipocytes in VAT and SAT of PHB Ad-KO male mice, compared to WT littermates. F: Ear-derived fibroblasts subjected to white adipogenesis for 8 days demonstrate accumulation of large lipid droplets (arrows) in PHB Ad-KO adipocytes. IF shows that PHB expression is lost in PHB Ad-KO adipocytes expressing perilipin-1 (PLN1). In all panels, plotted are mean \pm SEM; ${ }^{*} P<0.05$ (Student's t-test). Scale bar: $50 \mu \mathrm{m}$.

Figure 2- Lipid metabolism defect and insulin resistance in adipocyte PHB KO mice. A: Uptake of BODIPY- $\mathrm{C}_{16}$ FA (green) added to cell culture medium (2uM) by WT and PHB-null adipocytes non-stimulated or stimulated with insulin (200nM). B: QBT assay demonstrating that insulininduced LCFA uptake by PHB-null adipocytes is significantly lower than by WT adipocytes. N=5 wells. C: Triglyceride blood concentration analysis upon iv Intralipid infusion into pre-starved mice shows a delay in clearance for PHB Ad-KO littermates compared to WT $(\mathrm{N}=6)$. D: Respiratory exchange ratio (RER) measured by indirect calorimetry over 2 days demonstrates an increase in 
PHB Ad-KO male mice. E: Plasma concentration of FFA after isoproterenol injection, elevated at baseline and not further induced in PHB Ad-KO mice (N=5). F: IF on VAT and BAT sections reveals lower expression of phosphorylated hormone-sensitive lipase ( $\mathrm{pHSL}$ ) in adipocytes (expressing perilipin-1). G: Glucose tolerance test (GTT) in male mice raised on HFD. After o/n fasting, littermates $(\mathrm{N}=5)$ were injected with glucose $(1 \mathrm{~g} / \mathrm{kg}$ body weight) i.p. and glucose in blood was measured. $H$ : Insulin tolerance test (ITT) in male mice raised on HFD. After $4 \mathrm{hr}$ fasting, littermates $(\mathrm{N}=5)$ were injected with insulin $(0.6 \mathrm{U} / \mathrm{kg}$ body weight) i.p. and glucose in blood was measured. In all panels, plotted are mean \pm SEM; ${ }^{*} P<0.05$ (Student's t-test). Scale bar: $50 \mu \mathrm{m}$.

Figure 3- BAT adaptive thermogenesis defect in PHB Ad-KO mice. A: appearance of resected interscapular BAT in WT and PHB Ad-KO male and female littermates. B: H\&E-staining of sectioned BAT from WT and PHB Ad-KO littermates. Graph: adipocyte size quantification. C: Western blotting on extracts from WAT and BAT demonstrates the loss of UCP1 expression in BAT of PHB Ad-KO mice. Actin immunoblotting: loading control. D: Body temperature maintenance in mice placed at $4^{\circ} \mathrm{C}$, indicating reduced cold tolerance of PHB Ad-KO mice $(\mathrm{N}=5)$. E: Indirect calorimetry data showing reduced oxygen consumption by PHB Ad-KO mice. F: Earderived fibroblasts subjected to brown adipogenesis for 8 days demonstrate accumulation of larger lipid droplets (arrows) in PHB-KO adipocytes. G: IF on cells from $F$ shows that UCP1 expression (arrows) is not induced in PHB Ad-KO adipocytes expressing perilipin-1 (PLN1). H-I: XF Cell Mito Stress assay used to analyze mitochondrial respiration in cells from PHB Ad-KO and WT mice subjected to brown adipogenesis. Oxygen consumption rate (OCR), measured upon successive treatment with oligomycin, FCCP (Carbonyl cyanide-ptrifluoromethoxyphenylhydrazone) and rotenone / antimycin A, demonstrates lower basal and induced mitochondrial function in PHB-KO adipocytes. I: oligomycin-resistant OCR, reflecting ATP-uncoupled respiration, is lower in PHB-KO adipocytes. In all panels, plotted are mean \pm SEM; * $P<0.05$ (Student's t-test). Scale bar: $50 \mu \mathrm{m}$. 
Figure 4- PHB modulators suppress mitochondrial respiration and uncoupling.

A: Mouse brown preadipocytes were treated with indicated PHB ligands during brown adipogenesis induction for 8 days. Brightfield images demonstrate comparable adipocyte differentiation in control and treated cells. MitoTracker staining reveals lower mitochondrial activity in adipocytes upon PHB modulator treatment. Graph: red fluorescence quantification. Scale bar: $50 \mu \mathrm{m}$. B: Mouse brown preadipocytes were induced to undergo brown adipogenesis for 8 days and then treated with indicated PHB ligands. Seahorse XFe24 / Flux Assay demonstrates a decrease of mitochondrial respiration upon treatment with each PHB modulator. C: Analysis of data from $B$ revealing a decrease in basal and spare respiration and in uncoupling, but not in ATP production, upon treatment with each PHB modulator. Plotted are mean \pm SEM; ${ }^{*} P<0.05$ (Student's t-test). 

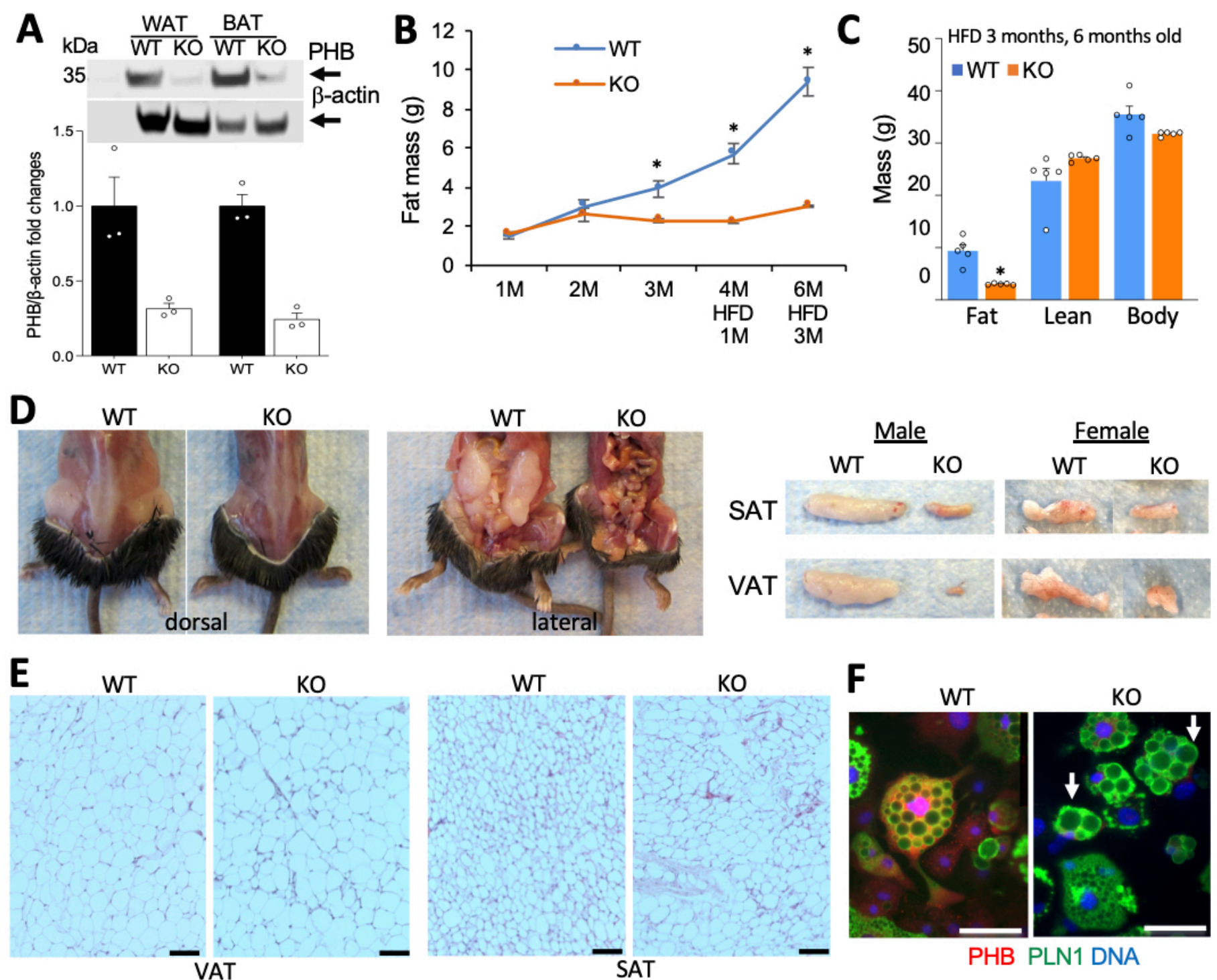

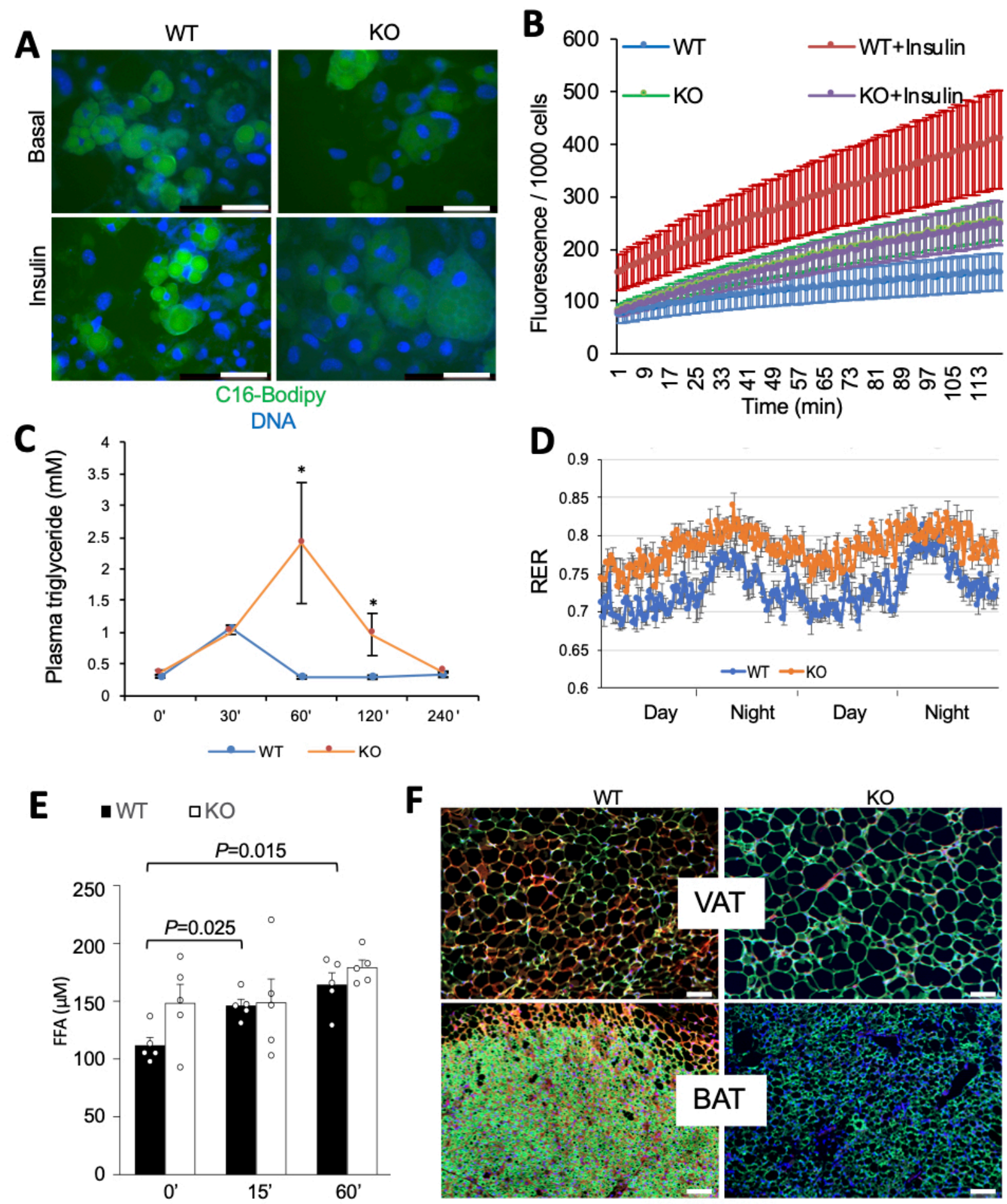

pHSL PLN1 DNA
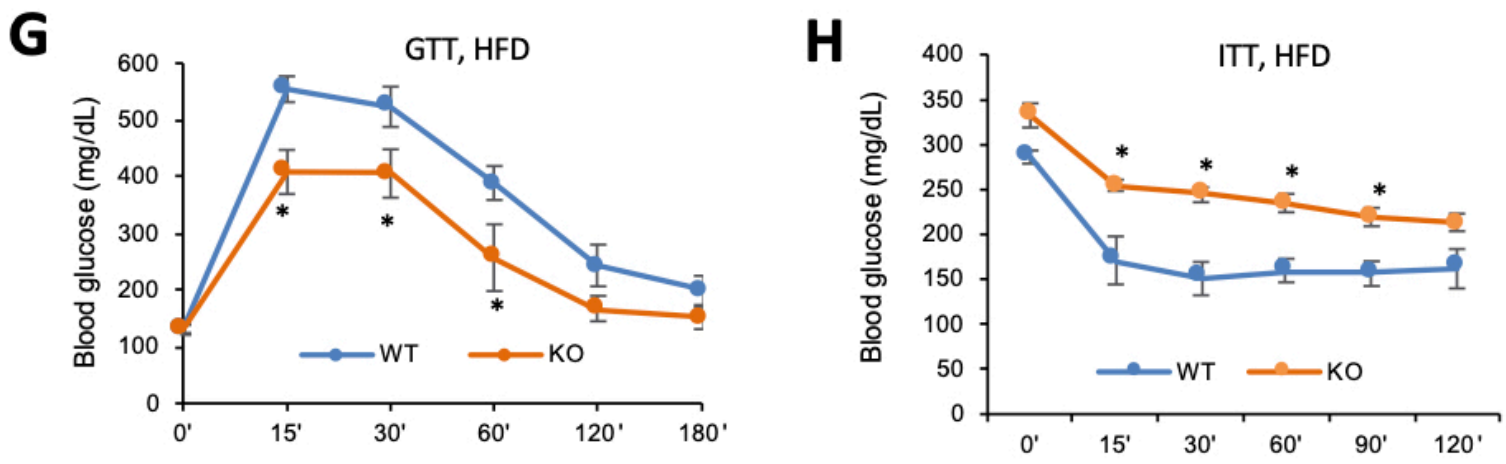

For Peer Review Only 


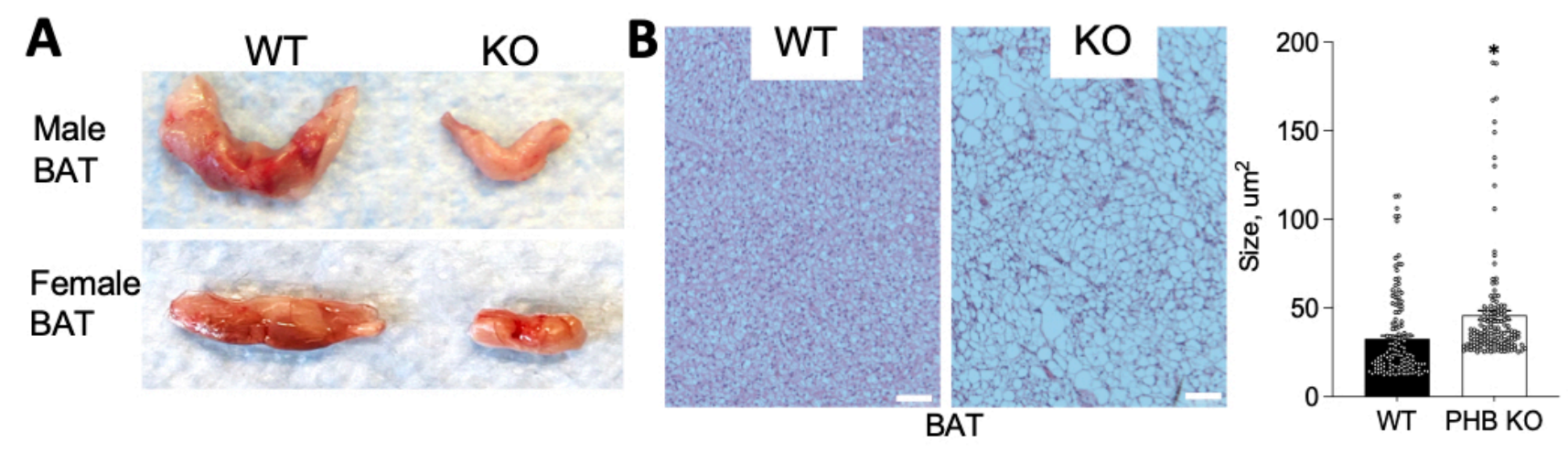

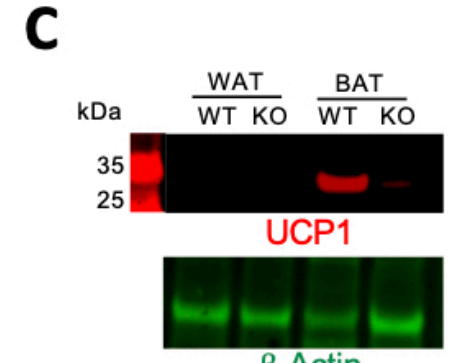

$\beta$-Actin
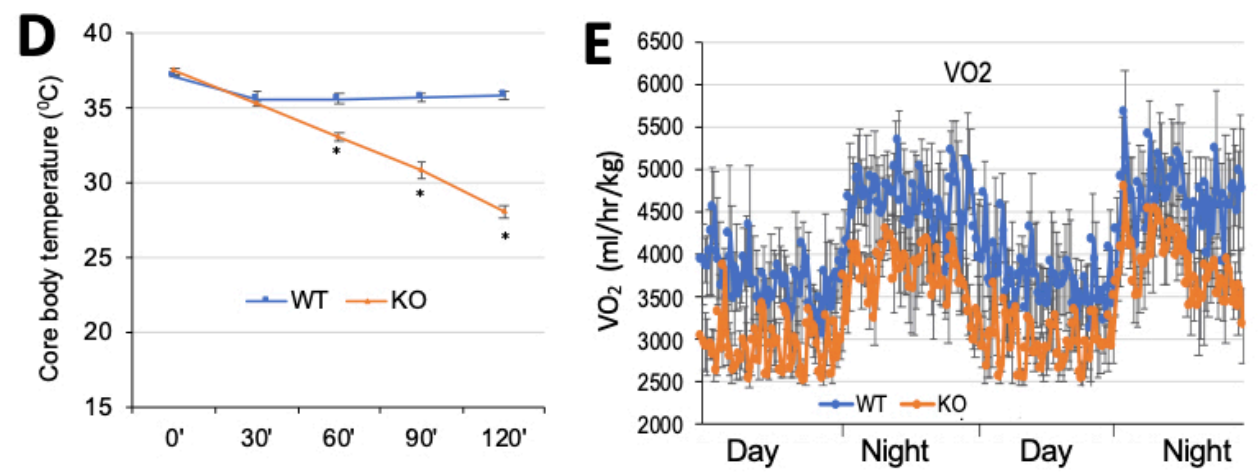

G
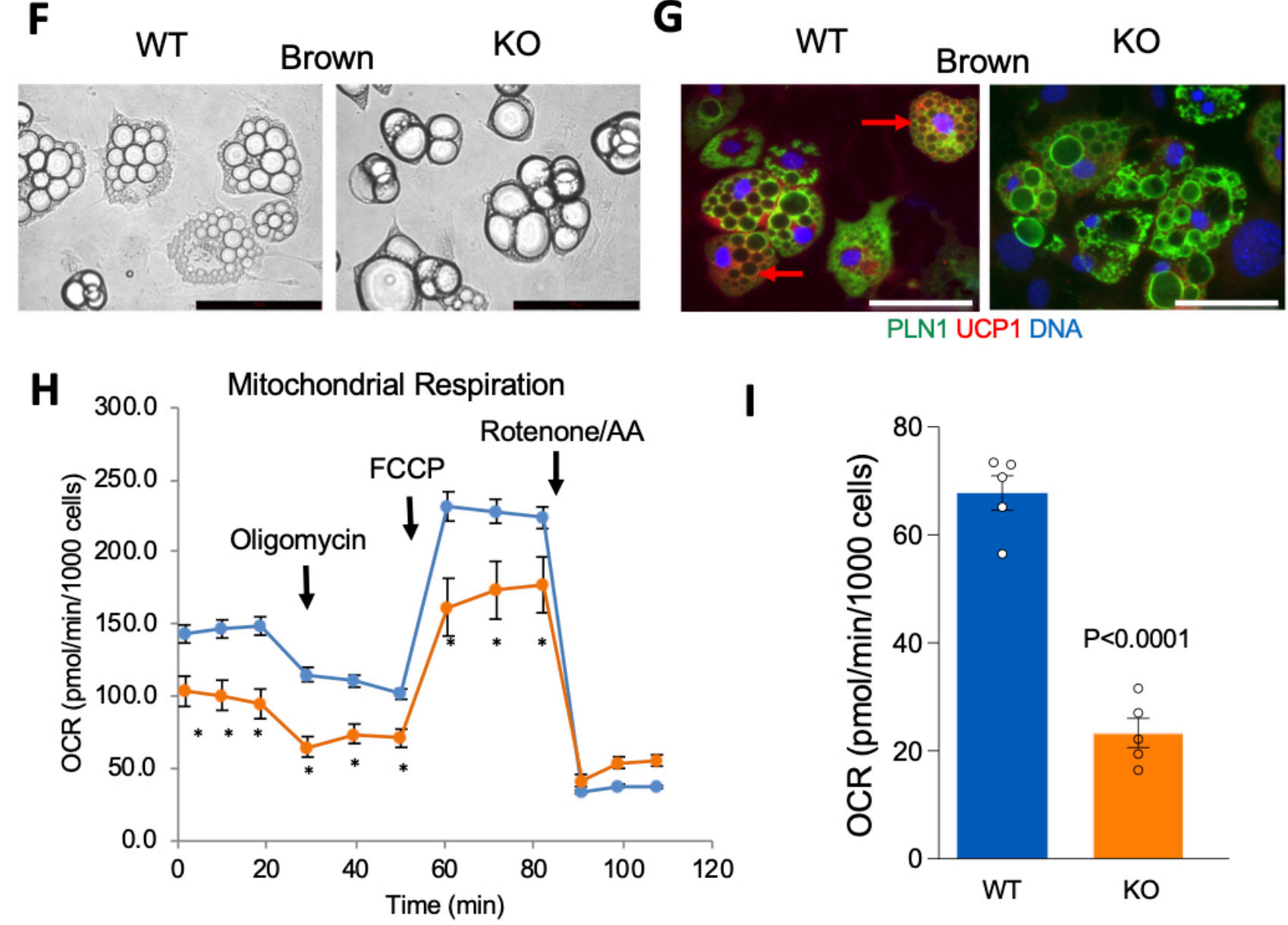


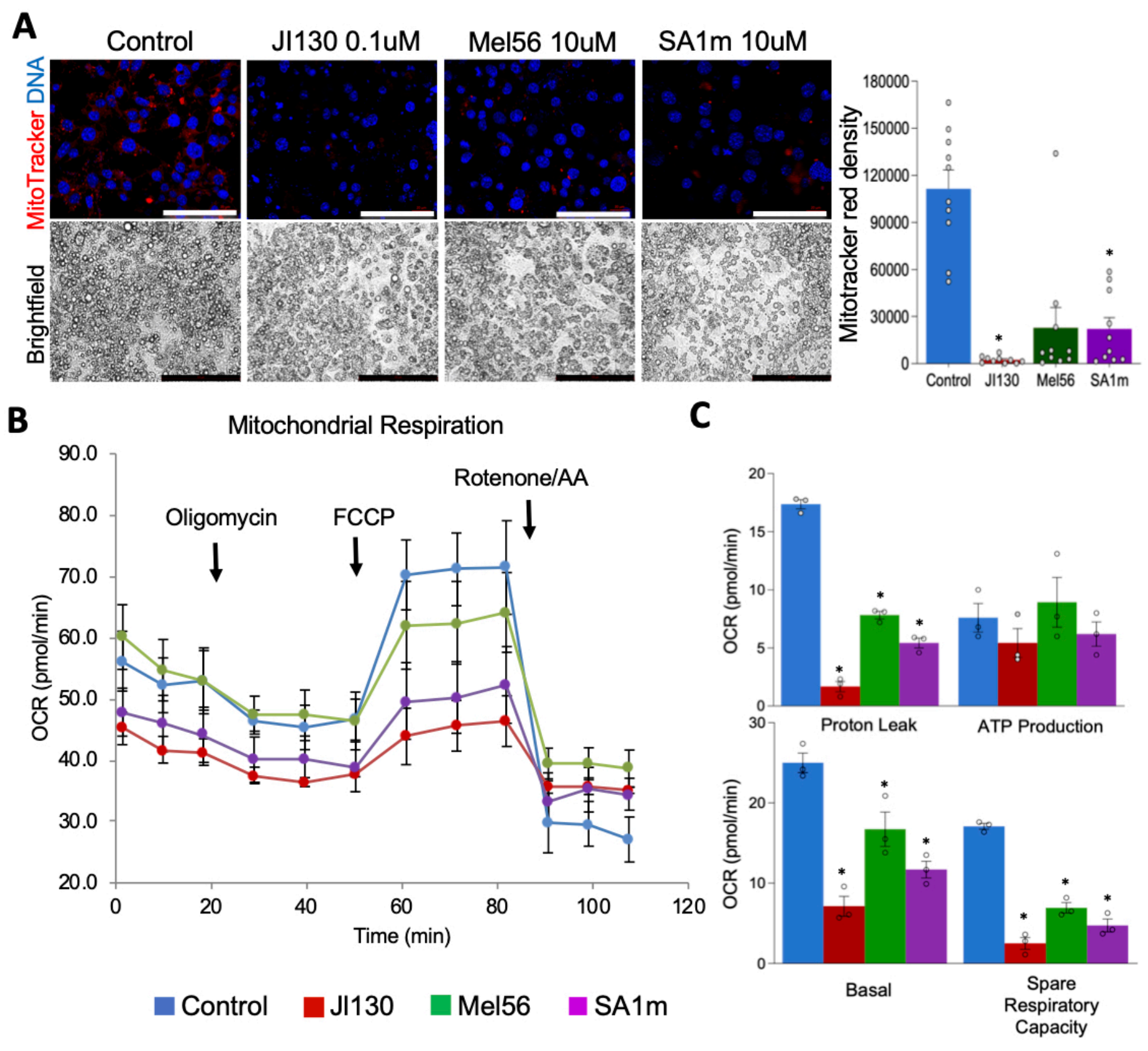


A
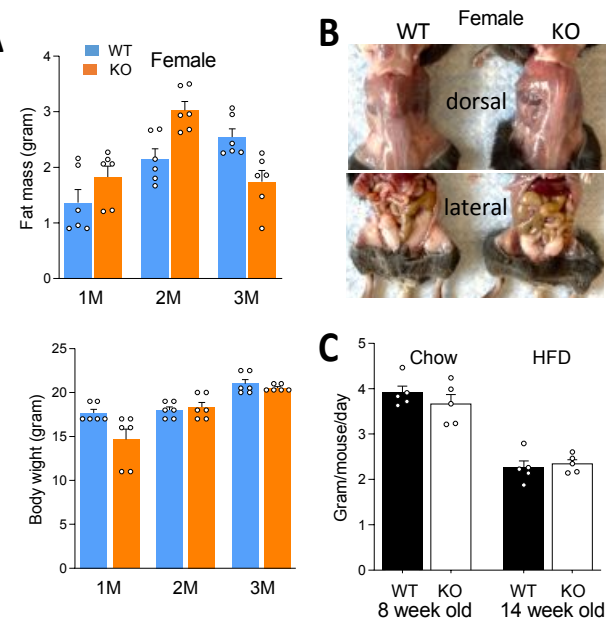

$\mathbf{F}$

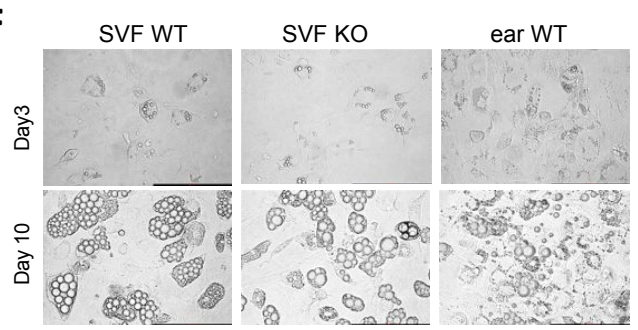

H

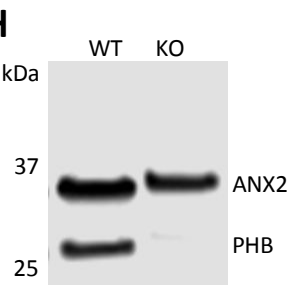

D
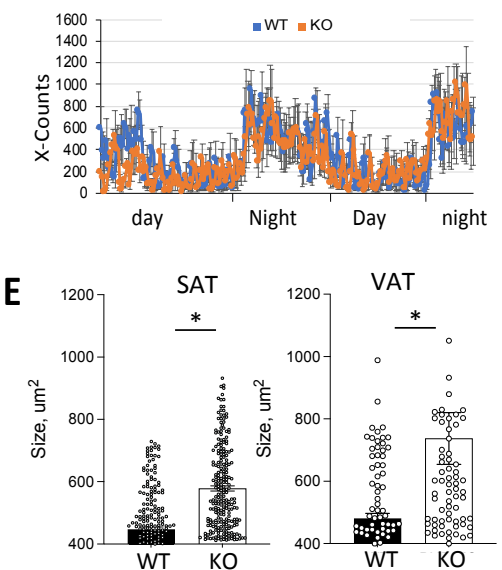

G

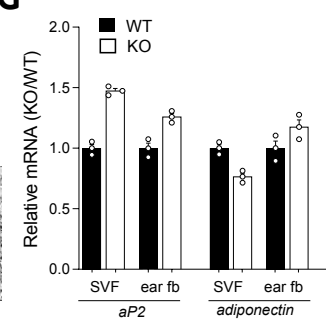

I

J WT $\underline{3 T 3-L 1}$ ко
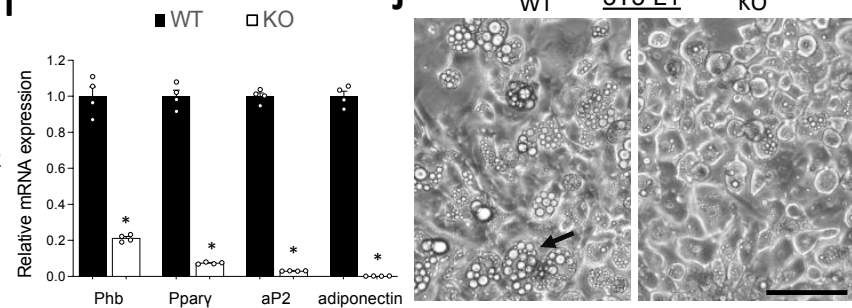

Figure S1- Adipocyte PHB KO mice display adipocyte abnormality.

$A$ : EchoMRI measurements on female mice raised on chow reveal a trend for reduced fat mass accumulation in PHB Ad-KO littermates detectable at 3 months. $\mathrm{N}=6$. B: Images demonstrating less subcutaneous and visceral AT in PHB Ad-KO female littermates. C: Food consumption measured over 2 days in PHB Ad-KO and WT littermates $(\mathrm{N}=5)$. $D$ : Spontaneous locomotor activity measured over 2 days in PHB Ad-KO and WT littermates $(\mathrm{N}=5)$. $E$ : Adipocyte size in males quantified. F: Brightfield microscopy on stromal cells derived from ears and SAT of WT and PHB Ad-KO subjected to adipogenesis. Arrows: lipid droplets, accumulating comparably in cells from WT and PHB-KO mice. G: RT-PCR for adipogenic genes reveals their similar expression in fibroblasts (fb) derived from SAT and ears of WT and PHB-KO mice 3 days post-induction. $H$ : Immunoblotting demonstrating Phb knockout in 3T3-L1 preadipocytes, ANX2 antibody was probed with for loading control. For CRISPR/Cas9 guide RNA expression, the 20-bp target sequence 5'-TCAATAAGCATGTCTCCGACTGG-3' containing the PAM sequence (underlined) was ligated into Lenti-CRISPR v2 plasmid (Addgene \#52961). I: RT-PCR for adipogenic genes reveals their reduced expression in Phb-KO 3T3-L1 adipocytes. Primers were as follows: Pparg: 5'- TTTAAAAACAAGACTACCCTTTACTGAAATT-3' and 5'AGAGGTCCACAGAGCTGATTCC-3'; Apn: 5'-TGTTCCTCTTAATCCTGCCCA-3' and 5'CCAACCTGCACAAGTTCCCTT-3'; aP2: 5'-GATGAAATCACCGCAGACGAC-3' and 5'ATTCCACCACCAGCTTGTCAC -3'. J: Lipid droplet formation (arrow) efficient in parental 3T3-L1 adipocytes but not in Phb-KO 3T3-L1 adipocytes. In all panels, plotted are mean \pm SEM. ${ }^{*} P<0.05$ (Student's t-test). Scale bar: $50 \mu \mathrm{m}$. 


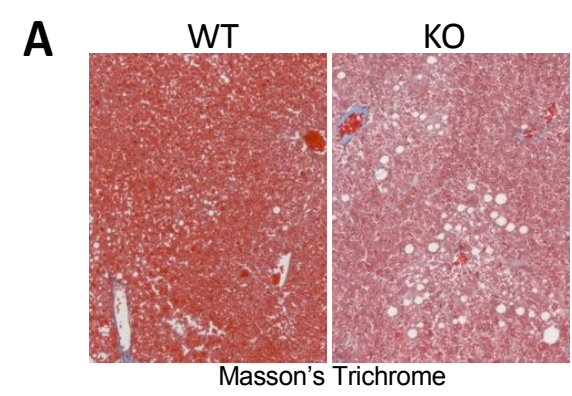

B

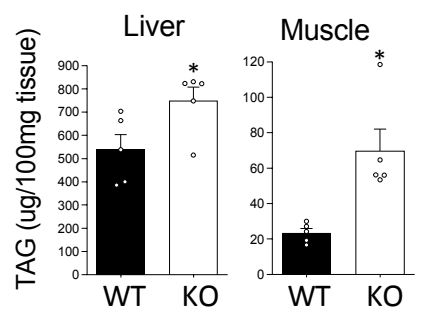

C SAT

VAT

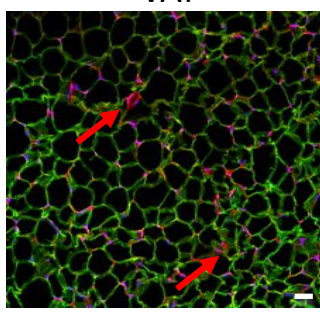

BAT
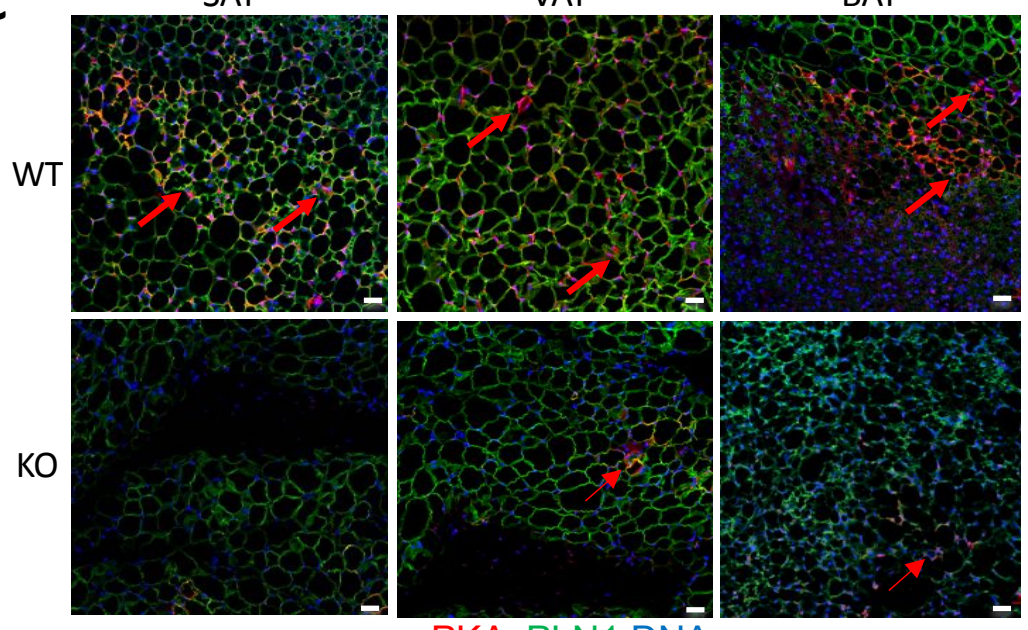

PPKA PLN1 DNA

Figure S2- Lipid metabolism disbalance in PHB Ad-KO mice.

A: Masson's trichrome staining reveals lipid droplet deposition in livers of PHB Ad-KO mice. B: Measurement of extracted triglycerides used for quantification of ectopic lipid deposition in livers and gastrocnemius muscle of Ad-KO mice. EnzyChrom ${ }^{\mathrm{TM}}$ Triglyceride Assay Kit from BioAssay System (ETGA-200) was used. C: IF analysis of PKA substrate phosphorylation (antibody from CellSignaling, Cat. \#9621, 1:100) in adipocytes of SAT, VAT and BAT identified based on perilipin-1 (PLN1) expression. Phospho-PKA (arrows) is lower in Ad-KO tissues. In all panels, plotted are mean \pm SEM. ${ }^{*} P<0.05$ (Student's t-test). Scale bar: $50 \mu \mathrm{m}$. 

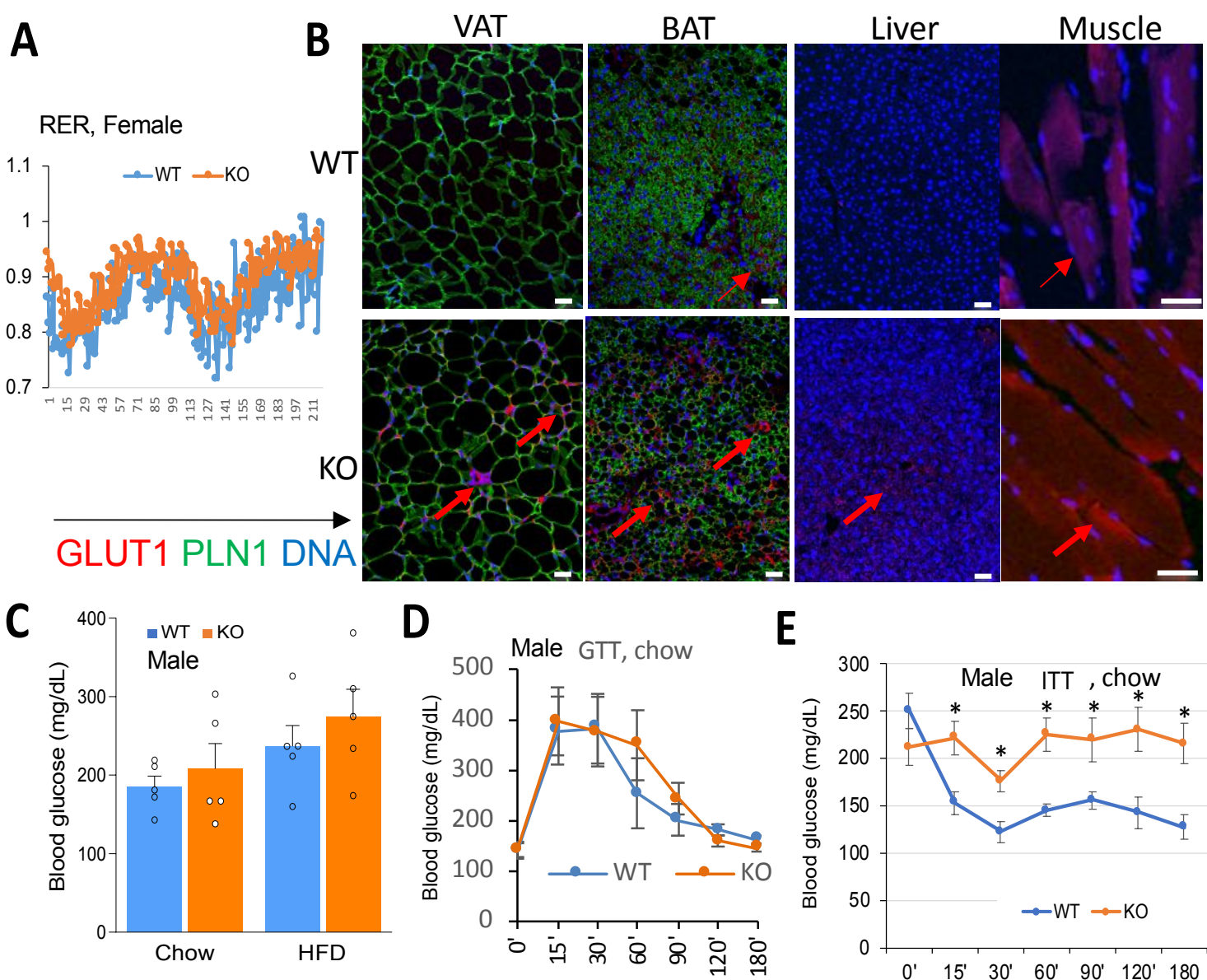

$\mathbf{F}$

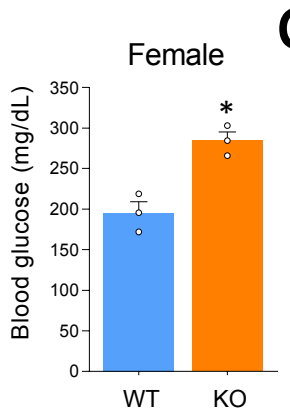

G

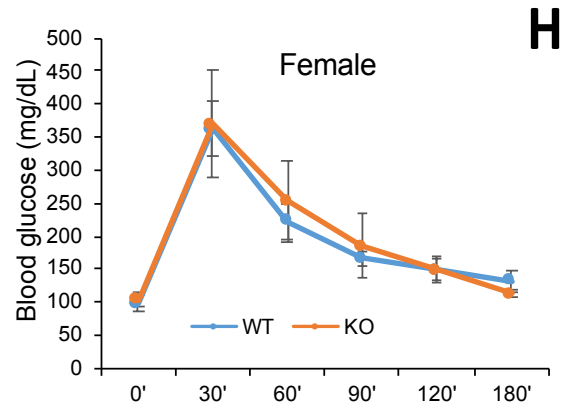

E

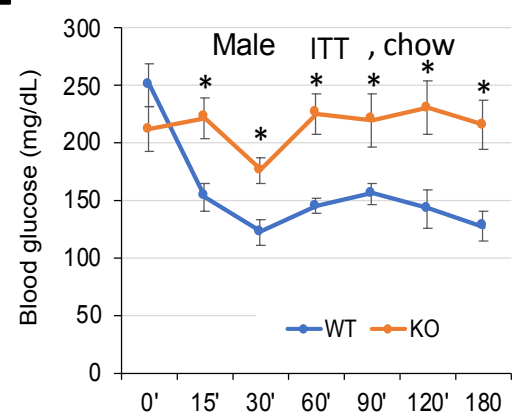

Figure S3- Metabolic phenotype of PHB Ad-KO males and females.

A: Respiratory exchange ratio (RER) shows an increase in PHB Ad-KO female mice. B: IF for GLUT1 (Antibody from Mybiosourse MBS179154, 1:100) demonstrates its increased expression in VAT, BAT, liver and muscle of PHB Ad-KO mice. C: Steady-state blood glucose levels in PHB Ad-KO and WT males. D: Glucose tolerance test in male mice raised on chow $(\mathrm{N}=5)$. $E$ : Insulin tolerance test (ITT) in male mice raised on chow ( $\mathrm{N}=5)$. F: Steady-state blood glucose levels in PHB Ad-KO and WT female littermates raised on chow. G: Glucose tolerance test in PHB Ad-KO and WT female littermates raised on chow ( $\mathrm{N}=5)$. $\mathrm{H}$ : Insulin tolerance test (ITT) in PHB Ad-KO and WT female littermates raised on chow $(\mathrm{N}=5)$. In all panels, plotted are mean $\pm \mathrm{SEM} ;{ }^{*} P<0.05$ (Student's t-test). Scale bar: $50 \mu \mathrm{m}$. 
A

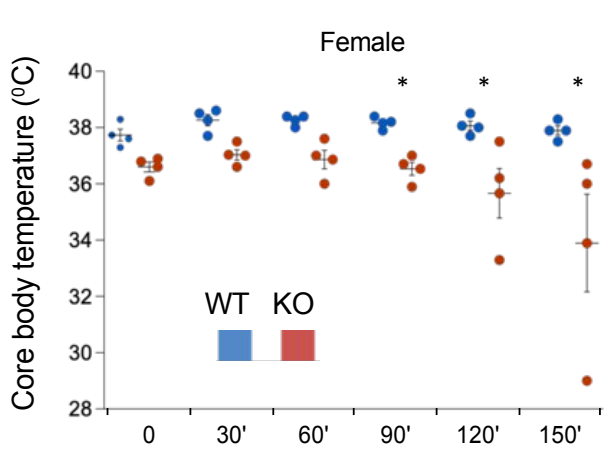

B

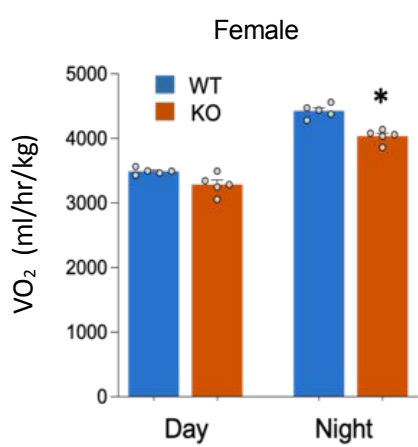

C
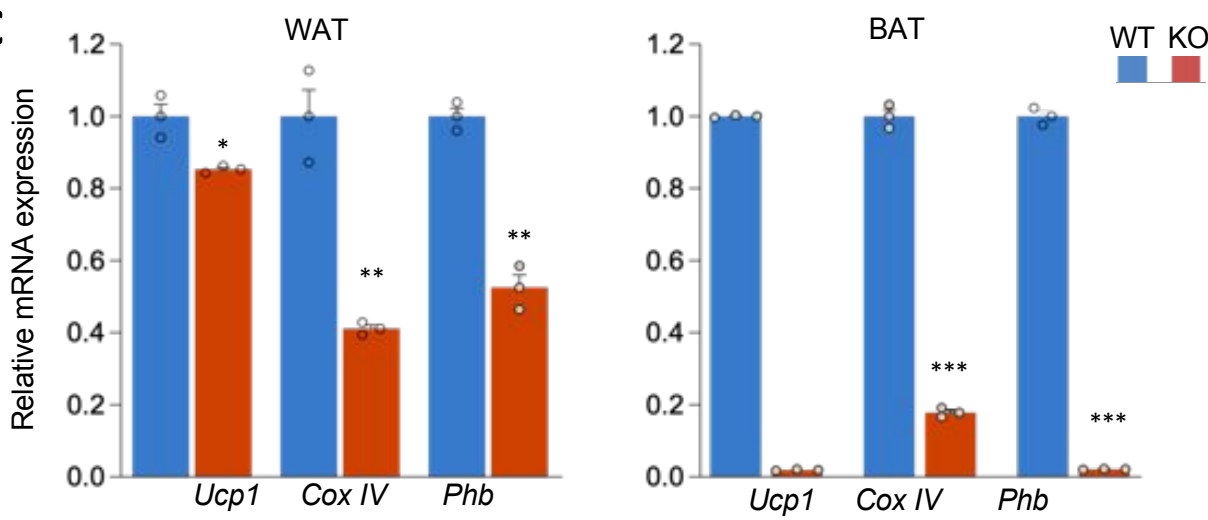

D

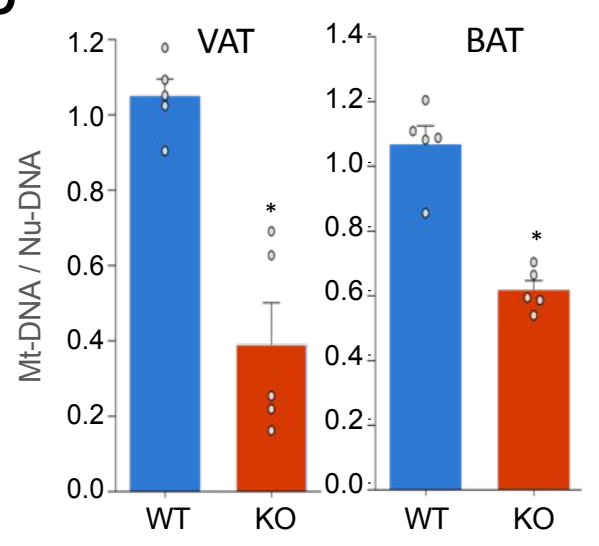

E

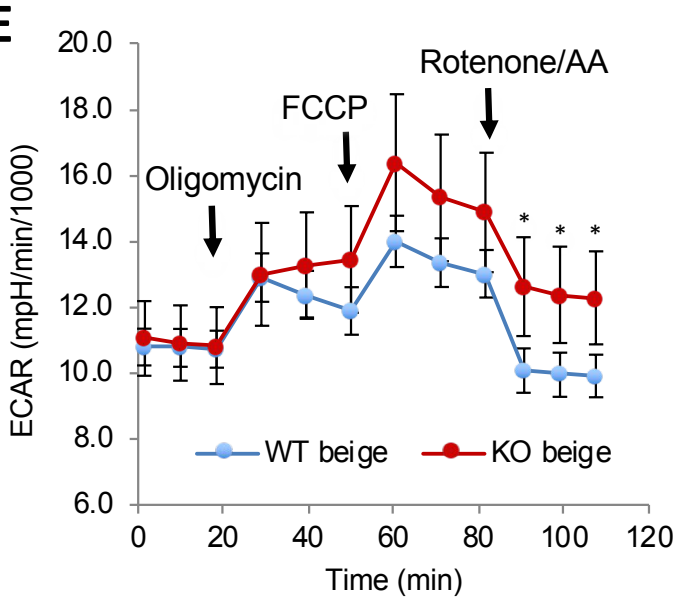

Figure S4- Mitochondrial dysfunction in BAT of PHB Ad-KO mice.

A: Reduced cold tolerance of female PHB Ad-KO mice at $4^{\circ} \mathrm{C}(\mathrm{N}=4)$. B: Indirect calorimetry data over 2 days showing reduced oxygen consumption by PHB Ad-KO female mice $(\mathrm{N}=4)$. C: Quantitative RTPCR analysis of Ucp1, Cox IV and Phb mRNA expression, normalized to 18S RNA, in WAT and BAT. Shown is a drastic decrease of Ucp1 and Cox IV in BAT of PHB Ad-KO littermates. D: RT-PCR with primer pairs specific for mitochondrial DNA and genomic DNA reveals a relatively low mitochondrial DNA content in both WAT and BAT of PHB Ad-KO mice ( $\mathrm{N}=5)$. Mouse mt-DNA 5'-GCC AGC CTG ACC CAT AGC CAT AAT AT-3' and 5'-GAG AGA TTT TAT GGG TGT AAT GCG G-3'; Nuc-DNA forward: 5'-TTG AGA CTG TGA TTG GCA ATG CCT-3' and 5'-CCT TTA ATG CCC ATC CCG GAC T-3'. E: Analysis of data from Fig. $3 \mathrm{H}$ on cells from PHB Ad-KO and WT mice. Extracellular acidification rate $(E C A R)$, measured upon successive treatment with oligomycin, FCCP (Carbonyl cyanide-ptrifluoromethoxyphenyl-hydrazone) and rotenone / antimycin A, demonstrates an increase in PHB-KO adipocytes. Plotted are mean \pm SEM; ${ }^{*} P<0.05$ (Student's t-test). 

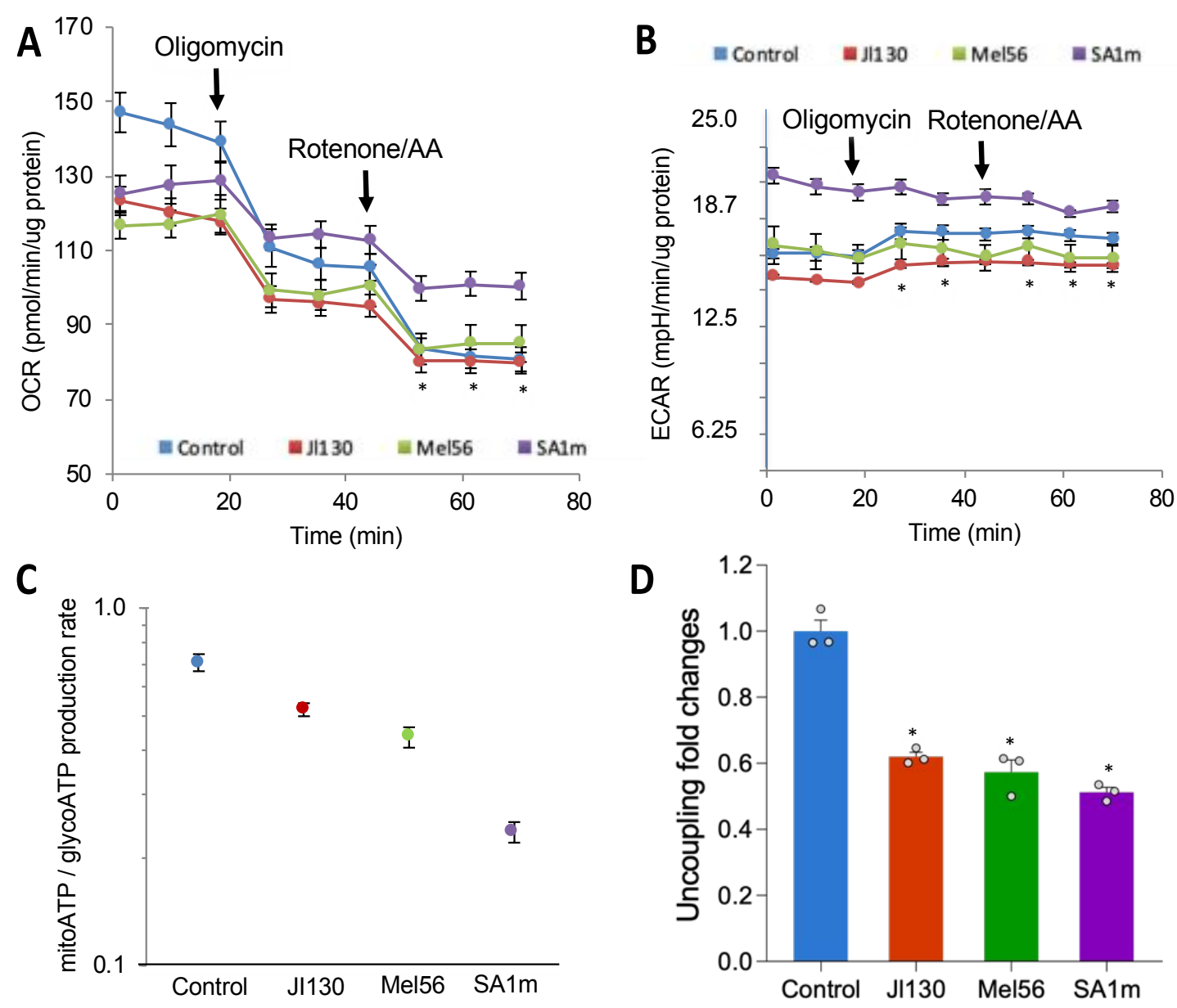

Figure S5- PHB modulators suppress mitochondrial respiration and uncoupling.

Mouse brown preadipocytes were treated with indicated PHB ligands during brown adipogenesis induction and subjected to Seahorse Real-time ATP Rate Assay on day 7. A: Decreased mitochondrial respiration in treated cells. $B$ : Decreased extracellular acidification in treated cells. C: XF ATP Rate Index indicating a shift to glycolytic ATP production in treated cells. D: Decreased ATP-uncoupled respiration in treated cells. Plotted are mean $\pm S E M ;{ }^{*} P<0.05$ (Student's t-test). 